\title{
Human Keratin 8 Variants Promote Mouse Acetaminophen Hepatotoxicity Coupled With c-Jun Amino-Terminal Kinase Activation and Protein Adduct Formation
}

\author{
Nurdan Guldiken, ${ }^{1,2}$ Qin Zhou, ${ }^{3}$ Ozlem Kucukoglu, ${ }^{2}$ Melanie Rehm, ${ }^{2}$ Kateryna Levada, ${ }^{1}$ Annika Gross, ${ }^{1}$ \\ Raymond Kwan, ${ }^{4}$ Laura P. James, ${ }^{5}$ Christian Trautwein, ${ }^{1}$ M. Bishr Omary, ${ }^{4}$ and Pavel Strnad ${ }^{1,2}$
}

Keratins 8 and $18(\mathrm{~K} 8 / \mathrm{K} 18)$ are the intermediate filaments proteins of simple-type digestive epithelia and provide important cytoprotective function. K8/K18 variants predispose humans to chronic liver disease progression and poor outcomes in acute acetaminophen (APAP)-related liver failure. Given that K8 G62C and R341H/R341C are common $\mathrm{K} 8$ variants in European and North American populations, we studied their biological significance using transgenic mice. Mice that overexpress the human K8 variants, $\mathrm{R} 341 \mathrm{H}$ or $\mathrm{R} 341 \mathrm{C}$, were generated and used together with previously described mice that overexpress wild-type K8 or K8 G62C. Mice were injected with $600 \mathrm{mg} / \mathrm{kg}$ of APAP or underwent bile duct ligation (BDL). Livers were evaluated by microarray analysis, quantitative real-time polymerase chain reaction, immunoblotting, histological and immunological staining, and biochemical assays. Under basal conditions, the $\mathrm{K} 8 \mathrm{G62C} /$ R341H/R341C variant-expressing mice did not show an obvious liver phenotype or altered keratin filament distribution, whereas $\mathrm{K} 8 \mathrm{G62C} / \mathrm{R} 341 \mathrm{C}$ animals had aberrant disulphide cross-linked keratins. Animals carrying the $\mathrm{K8}$ variants displayed limited gene expression changes, but had lower nicotinamide $\mathrm{N}$-methyl transferase (NNMT) levels and were predisposed to APAP-induced hepatotoxicity. NNMT represents a novel $\mathrm{K} 8 / \mathrm{K} 18$-associated protein that becomes up-regulated after $\mathrm{K} 8 / \mathrm{K} 18$ transfection. The more pronounced liver damage was accompanied by increased and prolonged JNK activation; elevated APAP protein adducts; K8 hyperphosphorylation at S74/S432 with enhanced keratin solubility; and prominent pericentral keratin network disruption. No differences in APAP serum levels, glutathione, or adenosine triphosphate levels were noted. BDL resulted in similar liver injury and biliary fibrosis in all mouse genotypes. Conclusion: Expression of human K8 variants G62C, R341H, or R341C in mice predisposes to acute APAP hepatotoxicity, thereby providing direct evidence for the importance of these variants in human acute liver failure. (HеPATOLOGY 2015;62:876-886)

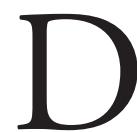
rug-induced liver injury represents a significant health problem given that it constitutes the major cause of acute liver failure (ALF) in the developed world and the major adverse event leading to termination of clinical drug development program. ${ }^{1,2}$ Acetaminophen (APAP) toxicity is the predominant cause of drug-induced ALF and accounts for approximately $50 \%$ of ALF cases in the United States and the

Abbreviations: ALF, acute liver failure; ALT, alanine aminotransferase; ALP, alkaline phosphatase; APAP, acetaminophen; ATP, adenosine triphosphate; BDL, bile duct ligation; GSSG/GSH, oxidized/reduced glutathione; h, human; IF, immunofluorescence; IP, immunoprecipitation; JNK, c-Jun amino-terminal kinase; K, keratin protein; $m R N A$, messenger RNA; NAPQI, N-acetyl-p-benzoquinone imine; NNMT, nicotinamide N-methyl transferase; PBC, primary biliary cirrhosis; P$J N K$, phosphorylated JNK; RT-PCR, real-time polymerase chain reaction; Tg, transgenic; TNF- $\alpha$, tumor necrosis factor alpha; WT, wild type.

From the ${ }^{1} I Z K F$ and Department of Internal Medicine III, University Hospital Aachen, Aachen, Germany; ${ }^{2}$ Department of Internal Medicine I, University Hospital Ulm, Ulm, Germany; ${ }^{3}$ Department of Medicine, Palo Alto VA Medical Center, Palo Alto, CA; ${ }^{4}$ Department of Molecular \& Integrative Physiology, University of Michigan Medical School, and the VA Ann Arbor Health Care System, Ann Arbor, MI; and ${ }^{5}$ Arkansas Children's Hospital Research Institute and Department of Pediatrics, University of Arkansas for Medical Sciences, Little Rock, AR

Received August 27, 2014; accepted May 3, 2015.

Additional Supporting Information may be found at onlinelibrary.wiley.com/doi/10.1002/hep.27891/suppinfo. 
UK. ${ }^{1}$ APAP is metabolized primarily through glucuronidation and sulfation whereas $5 \%-15 \%$ is oxidized through the cytochrome P450 system, thereby leading to production of $\mathrm{N}$-acetyl-p-benzoquinone imine (NAPQI).

At therapeutic APAP doses, NAPQI is detoxified by conjugation with sulfhydryl groups that are donated by glutathione. However, after higher exposure, glutathione becomes depleted and NAPQI binds covalently to hepatocellular proteins. This proteotoxic stress leads to activation of stress kinases, such as c-Jun-N-terminal kinase (JNK) and development of liver injury. ${ }^{3,4}$ While APAP is generally considered safe at therapeutic doses, nearly $30 \%$ of patients display an increase in alanine aminotransferase (ALT) levels after only 5 days of ingestion, thereby suggesting genetic differences in APAP susceptibility. ${ }^{3,5}$ Whereas genes responsible for APAP metabolism are the most obvious candidates, keratin polypeptides 8 and $18(\mathrm{~K} 8 / \mathrm{K} 18)$ are also candidate modifier genes that influence the outcome of APAPinduced ALF. ${ }^{3,6}$ In particular, a large study suggested that the presence of biologically significant $\mathrm{K} 8$ variants predisposes their carriers to an adverse outcome of APAP-induced ALF. ${ }^{6}$

Keratins represent the largest subgroup of the intermediate filament protein family and are expressed in all epithelia cells and skin appendages. ${ }^{7,8}$ Keratins are subdivided into type I (K9-K40) and II (K1-K8, K71$\mathrm{K} 86$ ), and both types assemble in an equimolar ratio to form obligate noncovalent heteropolymers. ${ }^{7,8}$ Keratins function as cytoprotective proteins and their mutations are associated with at least 40 different human diseases. ${ }^{9}$ Their heteropolymeric nature and epithelial cell-specific expression are reflected by the presence of cell-typeselective displays of keratin repertoires and pairings. ${ }^{7,10}$ For example, K8/K18 are the major keratins of singlelayered or glandular epithelia whereas the pairs, K1/K10 and $\mathrm{K} 5 / \mathrm{K} 14$, are expressed in suprabasal and basal epidermal cells, respectively. ${ }^{7,10}$

Adult hepatocytes are unique in that they express only K8/K18, whereas most other epithelial cells display a more complex keratin pattern, ${ }^{10,11}$ which explains why mutations in $\mathrm{K} 8 / \mathrm{K} 18$ result in a predominantly hepatic phenotype. Animals that lack K8/K18 or overexpress the keratin filament disruptor, K18 R90C, develop mild liver injury under basal conditions, but are markedly predisposed to injury in a wide range of experimental liver injury models. ${ }^{10-12}$ These animal findings led to human association studies that identified K8/K18 variants as risk factors for liver fibrosis and end-stage liver disease progression in several chronic liver disorders, such as hepatitis $\mathrm{C}$ infection and primary biliary cirrhosis (PBC). ${ }^{6,10}$ Moreover, K8 variants predisposed their carriers to an adverse acute liver injury outcome, as observed in patients with ALF. ${ }^{6}$ K8 G62C and K8 $\mathrm{R} 341 \mathrm{H}$ are the most common heterozygous $\mathrm{K} 8 / \mathrm{K} 18$ variants in Caucasians and are observed in 3.2\% and $1.5 \%$ of this population, respectively, whereas $\mathrm{K} 8$ $\mathrm{R} 341 \mathrm{C}$ is rare, but offers important insights into disease pathology owing to the toxicity of cysteines in simple epithelial keratins. ${ }^{6,12-14}$

Despite the large body of evidence linking K8/K18 variants to human liver disease, little is known regarding their underlying pathomechanisms. In vitro and in vivo cell culture and animal data, respectively, suggest that K8/K18 variants compromise stress-induced K8/K18 hyperphosphorylation and impair keratin network reorganization under these conditions. ${ }^{10,11,15,16}$ Similarly, livers of mice overexpressing K8 G62C stimulated with Fas ligand manifest markedly decreased K8 S74 phosphorylation and shunting of phosphorylation to other stress-activated kinase substrates with consequent enhanced liver injury, as compared with mice that overexpress WT K8. ${ }^{17}$

To determine what hepatic stresses are modulated by the presence of K8/K18 variants, and to study the underlying mechanisms, we generated mice that overexpress human $\mathrm{K} 8 \mathrm{R} 341 \mathrm{H}$ or $\mathrm{R} 341 \mathrm{C}$ and (together with the previously described $\mathrm{K} 8 \mathrm{G} 62 \mathrm{C}$ mice) subjected them to acute APAP injury or chronic biliary injury induced by bile duct ligation (BDL). Our data demonstrate that several naturally occurring $\mathrm{K} 8$ variants predispose to APAP toxicity through JNK activation, with increased K8 phosphorylation at S74 and S432, enhanced K8/K18 solubility and filament network disruption, as well as more-prominent formation of APAP-cysteine adducts.

\footnotetext{
This work was supported by the German Research Foundation grants STR 1095/2-1, STR 1095/4-1, the IZKF research group funding, and the SFB/TRR57 (to P.S. and C.T.), National Institutes of Health grant DK52951, and a Department of Veterans Affairs Merit Award (to M.B.O.).

Address reprint requests to: Pavel Strnad, M.D., Department of Internal Medicine III and IZKF, University Hospital Aachen, Pauwelsstraße 30, D-52074 Aachen, Germany. E-mail: pstrnad@ukaachen.de; fax: +49(241) 80-82455.

Copyright $(2015$ by the American Association for the Study of Liver Diseases.

View this article online at wileyonlinelibrary.com.

DOI 10.1002/hep.27891

Potential conflict of interest: Dr. James owns stock and received grants from Acetaminophen Toxicity Diagnostics.
} 


\section{Materials and Methods}

Mouse Experiments. Transgenic (Tg) mice that overexpress human (h) K8 R341H/R341C were generated as previously described for the mice that overexpress human K8 G62C. ${ }^{17}$ First, a BamHI-SalI fragment from the wild-type (WT) 12-kilobase genomic sequence $^{18}$ was subcloned into pcDNA3.1 vector. The Arg-to-His/Cys mutation (CGT->CAT/TGT) was introduced using a Quickchange site-directed mutagenesis kit (Stratagene, La Jolla, CA) and confirmed by sequencing. The mutated $\mathrm{K} 8$ fragment was complemented with a K8 segment excised from the original clone. The constructs were injected into pronuclei of fertilized FVB/n mouse eggs. The offspring was genotyped with primers specific for hK8, as described previously $^{17}$ (Supporting Table 1) and maintained on an $\mathrm{FVB} / \mathrm{n}$ background. To confirm the presence of the described K8 variants, sequencing of K8 exons 1 and 6 was performed (Supporting Fig. 1; Supporting Table 1). The strains used in this study are termed as WT K8, K8 G62C, and K8 R341H/C mice to highlight the K8 variant they overexpress. Notably, the sequence identity of mouse and human $\mathrm{K} 8$ is $88 \%$, and the K8 G62 and R341 residues are highly conserved across species. In addition, we analyzed the previously described K18-null mice $^{19}$ and their non-Tg littermates (both are in an FVB/N background).

To study APAP hepatotoxicity, 2-month-old mice were fasted overnight, injected intraperitoneally with APAP $(600 \mathrm{mg} / \mathrm{kg}$ mouse weight; Fagron GmbH\&Co KG, Barsbüttel, Germany) and sacrificed 4 or 18 hours later by $\mathrm{CO}_{2}$ inhalation followed by blood collection. In another set of experiments, BDL was performed in 3month-old animals anesthetized with isoflurane. After midline laparotomy, the bile duct was ligated with 2-0 or 5-0 nonabsorbable surgical silk (Perma-hand; Ethicon, Somerville, NJ) and animals were followed for 21 days. Dissected livers were weighed and pieces were fixed using 10\% formaldehyde (histological staining), snap frozen in liquid nitrogen (biochemical analysis), or placed in RNAlater stabilization reagent (Ambion, Life Technologies GmbH, Darmstadt, Germany). Serum parameters were measured in the Clinical Chemistry Department (University Hospital Ulm, Ulm, Germany). Animal experiments were approved by the institutional animal care committees of the participating centers. For details on staining, messenger RNA (mRNA) isolation, real-time polymerase chain reaction (RT-PCR), microarray analysis, biochemical methods, cell transfection experiments, and statistical evaluation, see the Supporting Methods section.

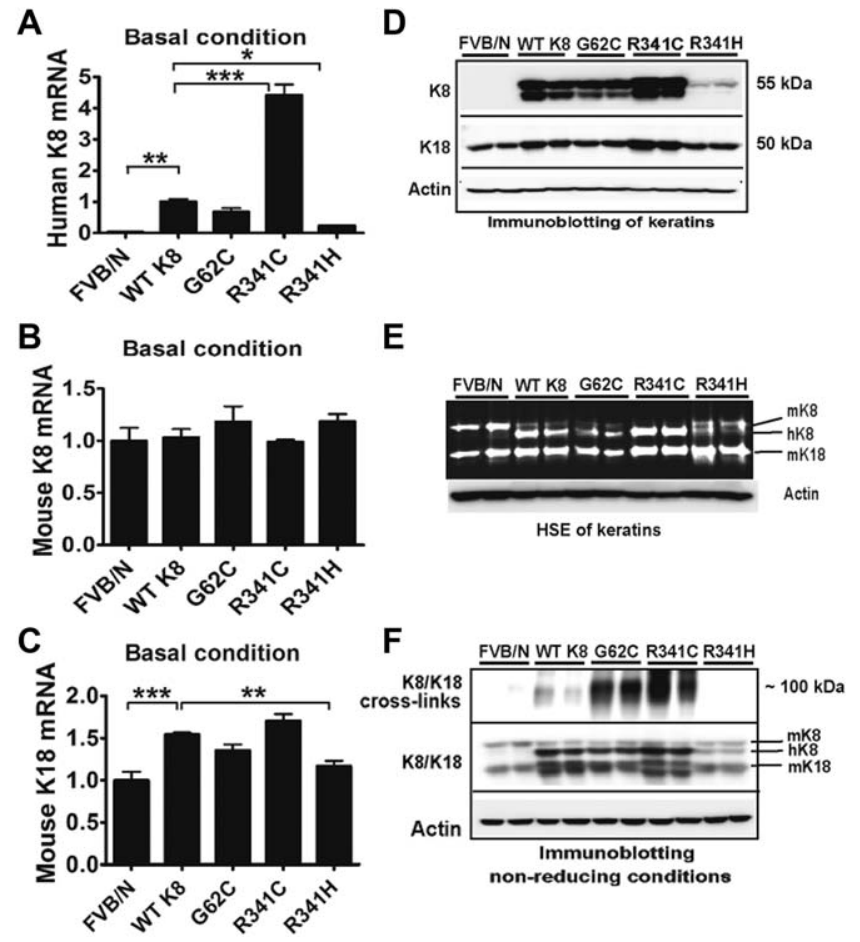

Fig. 1. Keratin $8 \mathrm{G} 62 \mathrm{C} / \mathrm{R} 341 \mathrm{C}$ variants promote keratin crosslinking under basal conditions. Human (h) K8 (A) and mouse (m) K18/K8 (B and C) mRNA and protein levels (D) were quantified by RT-PCR and immunoblotting, respectively. (E) To directly visualize proteins, a high salt extraction (HSE) with SYPRO ruby staining was performed from non-Tg livers (FVB/N) as well as tissues overexpressing WT K8 or the highlighted $\mathrm{K} 8$ variants. (F) To test the formation of disulfide bridges, livers were homogenized under nonreducing conditions. After longer exposure (upper panel), a significant amount of cross-linked hK8 was detected in G62C and R341C livers and the cross-links went away under reducing conditions (not shown). L7 (mouse ribosomal protein) and actin were used as an internal/loading control for quantitative RTPCR and immunoblotting, respectively. At least 4 mice were analyzed per genotype and the results are expressed as mean \pm standard error of the mean. ${ }^{*} P<0.05 ;{ }^{* *} P<0.01 ;{ }^{* * *} P<0.001$.

\section{Results}

To characterize the $\mathrm{Tg}$ mouse lines generated in this study, we first quantified their keratin expression levels. All Tg lines displayed moderate hK8 mRNA overexpression, with levels being K8 R341C $>$ WT K8 K8 $\mathrm{G} 62 \mathrm{C} \gg \mathrm{K} 8 \mathrm{R} 341 \mathrm{H}$ whereas no changes in mouse $(\mathrm{m})$ K8 mRNA were observed (Fig. 1A,B). Consistent with a previous report, ${ }^{20} \mathrm{~K} 8$ overexpression up-regulated K18 mRNA levels, and, consequently, the highest K18 mRNA was observed in WT K8 and K8 R341C mice (Fig. 1C). hK8 and K18 protein levels mirrored the mRNA results, whereas hK8 overexpression led to compensatory downregulation of $\mathrm{mK} 8$ (Fig. 1D,E), which is in line with previous reports of WT K8 overexpression. ${ }^{17,20}$

Given that introduction of cysteine residues into otherwise cysteine-free hK8 may lead to formation of disulphide bridges, ${ }^{14,17}$ we analyzed K8/K18 species under 
nonreducing conditions. High-molecular-weight K8/ K18 species were easily observed in K8 G62C/R341C micebut not in WT K8 and K8 R341H animals (Fig. $1 \mathrm{~F})$. All $\mathrm{Tg}$ mouse lines displayed a normal-appearing $\mathrm{K} 8 / \mathrm{K} 18$ filament network in the liver under basal conditions; however, this network was more dense in the K8 R341C livers that carry the highest K8 expression levels (Fig. 2; Supporting Fig. 2).

hK8 overexpression did not cause any lethality, growth defect, or organ malfunction (not shown). Under basal conditions, all Tg lines had normalappearing liver architecture and normal ALT levels (Fig. $3 \mathrm{Aa}-\mathrm{d}, \mathrm{B})$. To further characterize the consequences of the presence of $\mathrm{K} 8$ variants, we performed microarray analysis that revealed modest changes among the genotypes (Fig. 4 and Supporting Fig. 3; Supporting Tables 2 and 3). Notably, nicotinamide N-methyltransferase (NNMT) was the only mRNA that was significantly altered in all mouse lines harboring $\mathrm{K} 8$ variants (Fig. 4D). Interestingly, K8/K18 transfection into NIH-3T3 cells resulted in NNMT up-regulation, whereas K18null livers and their normal controls displayed similar NNMT expression levels (Supporting Fig. 4). In addition, immunoprecipitation (IP) experiments in two different hepatocellular cell lines (HepG2 and Huh7) showed coimmunoprecipitation of NNMT with K8/ K18 (Fig. 4E-F).

Given that NNMT represents a prominent Nmethylating enzyme involved in xenobiotic metabolism $^{21}$ and given that patients carrying $\mathrm{K} 8$ variants are predisposed to APAP-induced ALF, ${ }^{6}$ we examined the susceptibility of our animals to this form of injury. Four hours post-APAP administration, mild hepatocellular swelling and elevated ALT levels were noted; however, both parameters did not differ significantly among the genotypes (Fig. 3Ae-h,C). More-prominent hepatocyte swelling and centrolobular necrosis became apparent 18 hours post-APAP challenge, and both liver damage and ALT levels were significantly higher in mice with K8 variants, compared to WT K8 mice (Fig. 3Ai-l,D,E). On the other hand, no obvious differences in susceptibility to APAP injury were noted between WT K8 mice and their non-TG littermates (not shown) or between WT and K18 KO animals (Supporting Fig. 5).

To delineate the mechanisms leading to increased APAP hepatotoxicity in mice with $\mathrm{K} 8$ variants, we examined APAP metabolism. Neither APAP plasma levels (4 hours post-APAP administration) nor expression of APAP-metabolizing enzymes differed significantly among the Tg mouse lines (Supporting Figs. 6 and 7). Notably, 18 hours post-APAP administration, APAP plasma levels were below the detection limit in all

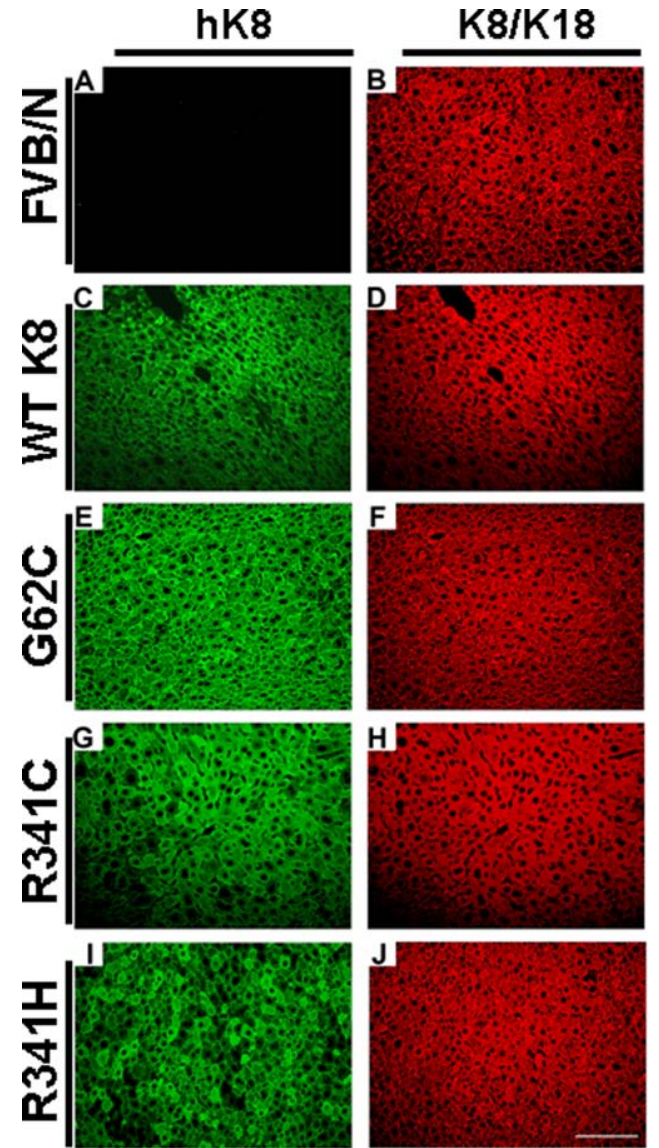

Fig. 2. Overexpression of $\mathrm{K} 8$ variants does not alter the morphology of keratin network under basal condition. Livers from Tg mice overexpressing WT human (h) keratin 8 (WT K8), hK8 G62C, or K8 R341C/ $\mathrm{R} 341 \mathrm{H}$ variant, and from non-Tg animals (FVB/N) were stained with an antibody recognizing both mouse and human K8/K18 (antibody 8592) or an antibody specific for hK8 (M20). Only nonspecific, weak hK8 staining was observed in FVB/N animals (A) whereas K8 R341H livers displayed a somewhat patchy hK8 distribution (I). Scale bar $=$ $100 \mu \mathrm{m}$.

genotypes (not shown). Next, we evaluated glutathione content because its depletion represents a crucial event in APAP toxicity. ${ }^{3,4}$ Under basal conditions, all animals displayed similar total glutathione levels and oxidized/ reduced glutathione GSSG/GSH ratios (Supporting Fig. 8A,B). As expected, APAP treatment resulted in depletion of total glutathione and an increase in the GSSG/GSH ratio, but both parameters were similar across genotypes (Supporting Fig. 8A,B).

APAP administration also leads to hepatic inflammation with increased plasma interleukin- 6 and tumor necrosis factor alpha (TNF- $\alpha$ ) levels as well as adenosine triphosphate (ATP) depletion. ${ }^{3,4}$ However, comparable cytokine and ATP levels were observed in all mouse lines pre- and post-APAP exposure (Supporting Fig. 8C-E). In addition, apoptosis levels post-APAP exposure were negligible (as expected ${ }^{4}$ ) and did not differ significantly among genotypes (Supporting Fig. 8F). 
A
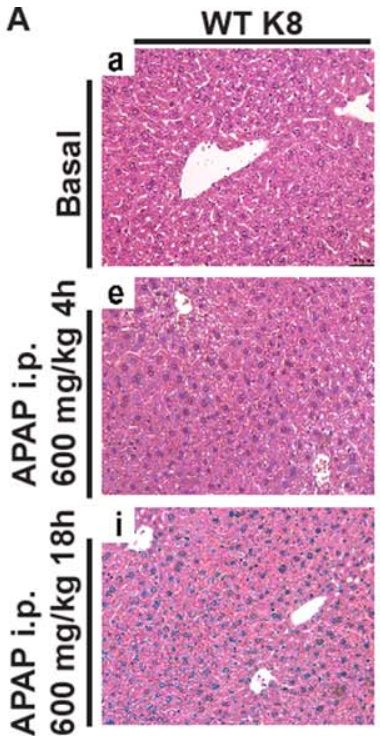

B

Basal condition

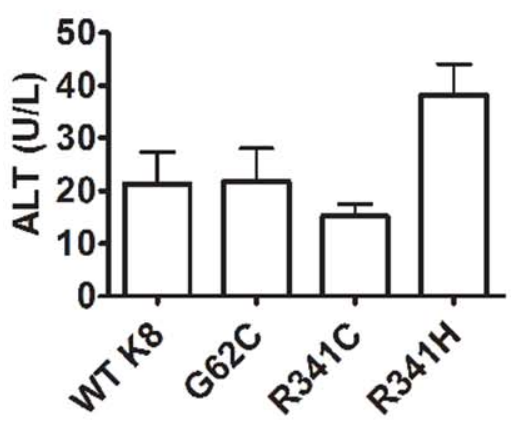

D

\section{APAP-600 $\mathrm{mg} / \mathrm{kg} 18 \mathrm{~h}$}

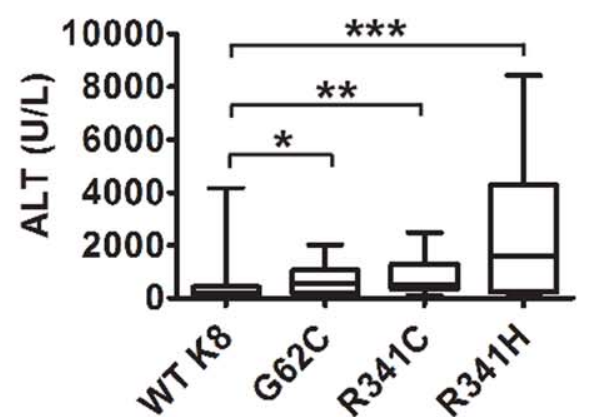

G62C
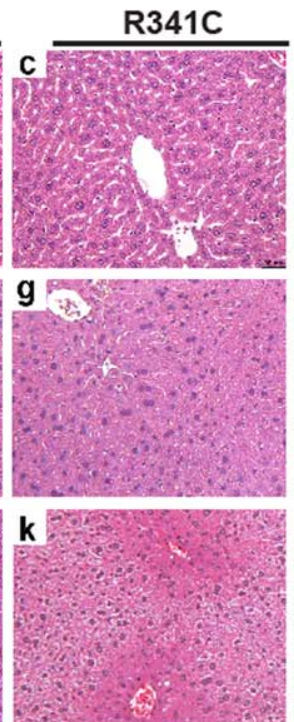

C
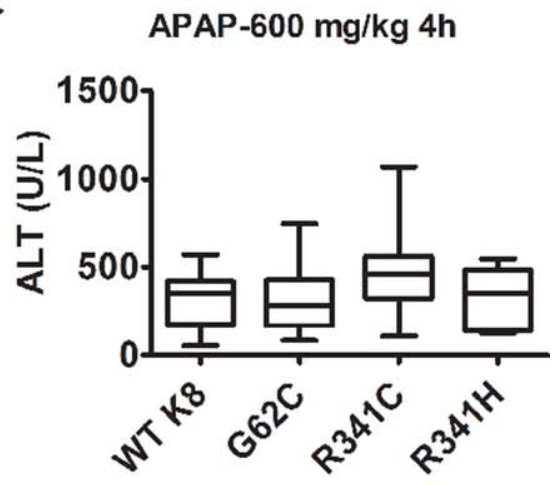

E

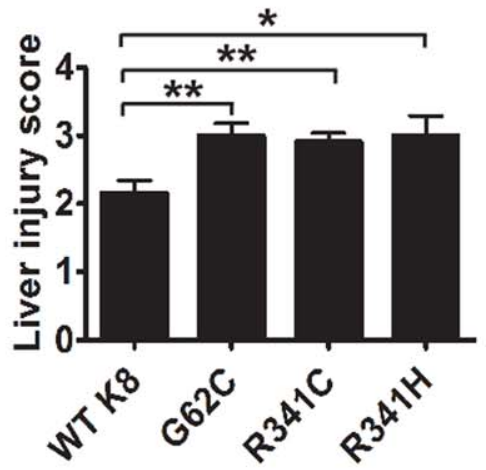

Fig. 3. K8 G62C and R341C/H variants promote APAP-induced liver injury. Mice overexpressing WT K8 or the K8 G62C/R341H/R341C variant were injected with $600 \mathrm{mg} / \mathrm{kg}$ of APAP intraperitoneally (i.p.) and sacrificed after 4 and 18 hours, respectively. (A) H\&E staining detected no obvious liver injury in untreated animals (a-d), whereas only a mild hepatocellular swelling that did not differ among the genotypes was noted 4 hours post-APAP exposure (e-h). Distinct hepatocyte swelling and centrolobular necrosis became apparent 18 hours post-APAP challenge (i-I) and the liver damage was more prominent in mice carrying K8 variants, as compared to WT K8, as quantified by morphometric analysis (E). Largely normal ALT levels were observed in untreated mice (B) whereas animals exposed to APAP for 4 hours displayed uniformly elevated ALTs that did not differ among genotypes (C). (D) Eighteen hours post-APAP administration, mice carrying K8 variants displayed significantly higher ALT levels than WT K8 animals (medians: WT K8: $221 \mathrm{U} / \mathrm{L} ; \mathrm{K} 8$ G62C: $554 \mathrm{U} / \mathrm{L} ; \mathrm{K} 8 \mathrm{R} 341 \mathrm{C}: 485 \mathrm{U} / \mathrm{L} ; \mathrm{K} 8$ R341H: 2,165 U/L). Box plots display median with first and third percentile, whereas whiskers indicate smallest and largest nonoutlier observations. For APAP experiments, at least 10 mice were analyzed per genotype. In $(E)$, the results are expressed as mean \pm standard error of the mean. Scale bar $=100 \mu$ m. ${ }^{*} P<0.05$; $* * P<0.01 ; * * * P<0.001$. Abbreviation: $\mathrm{H} \& \mathrm{E}$, hematoxylin and eosin. 
A

\begin{tabular}{ccc}
\hline Gene & \multicolumn{2}{c}{ Fold change } \\
\cline { 2 - 3 } name & G62C/WT K8 & R341H/WT K8 \\
\hline Nnmt & $-1,8$ & $-4,3$ \\
Ren2 & $-2,4$ & $-2,2$ \\
Dpt & $+1,9$ & $+1,6$ \\
Got1 & n.s & $-1,9$ \\
Gck & n.s & $+1,9$ \\
Car3 & n.s & $+2,6$ \\
Cyp7a1 & $-2,6$ & n.s \\
Idil & $-2,2$ & n.s \\
Tubb2a & $+1,7$ & n.s \\
Hspa1a & $+2,2$ & n.s
\end{tabular}

B

C
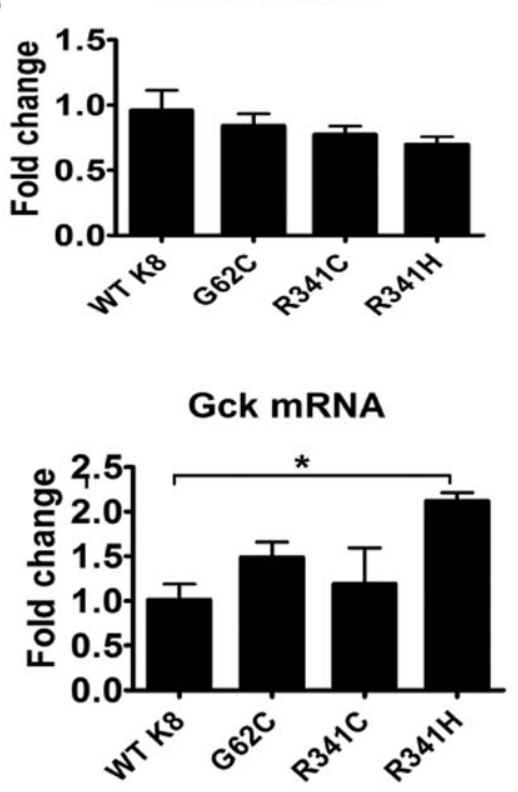

D

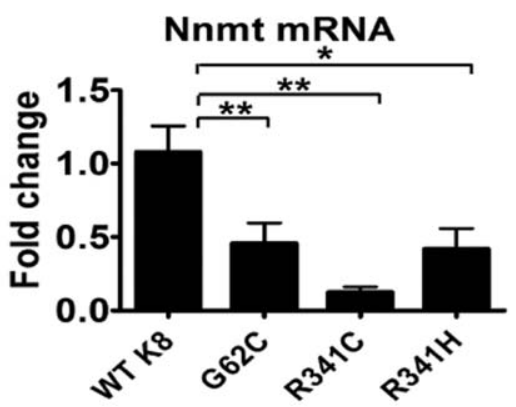

E
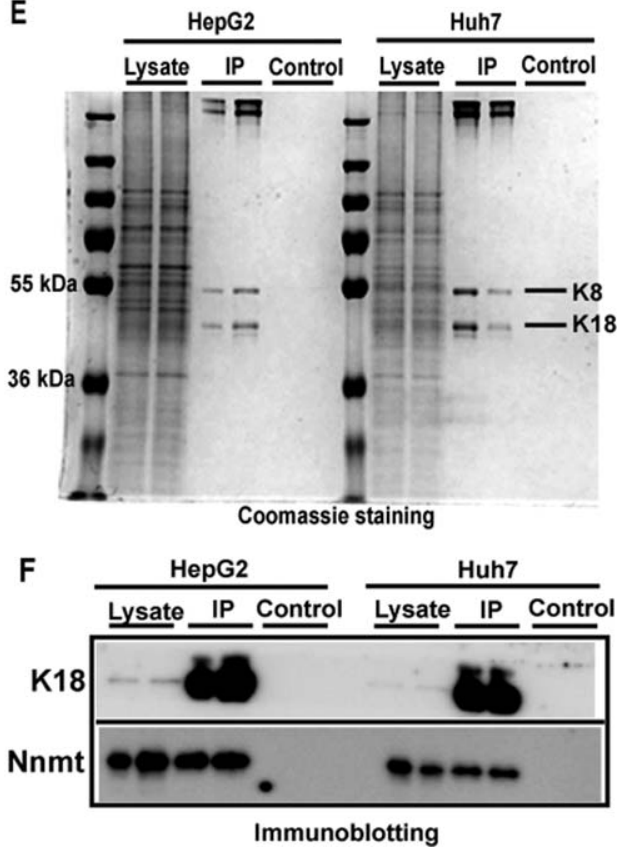

Fig. 4. NNMT represents a novel K8/K18-associated protein that was discovered by microarray analysis. (A) List of genes that are most significantly altered in untreated mice carrying K8 G62C/R341H variant versus animals overexpressing WT K8, as determined by expression profiling analysis. RT-PCR analysis (B-D) demonstrated that the drug metabolism-associated gene, NNMT, is the only gene that is similarly reduced in all mouse lines carrying K8 variants. L7 (mouse ribosomal protein) gene was used as an internal control. At least 4 mice were analyzed per genotype and results are expressed as mean \pm standard error of the mean. ${ }^{*} P<0.05$; ${ }^{* *} P<0.01$. (E and $\mathrm{F}$ ) To immunoprecipitate K8/K18, $1 \%$ NP-40 lysates from HepG2 or Huh7 cells were incubated with protein G dynabeads conjugated with the anti-human K18 antibody, L2A1 (IP). Nonconjugated beads were used as a control. The lysates before incubation with the antibody were analyzed as an input. (E) Coomassie staining confirms the specificity of the IP, whereas immunoblotting for the indicated antigens (F) identifies NNMT as a K8/K18-associated protein. Abbreviation: n.s., not significant.

We analyzed the levels of total JNK1 and JNK2, the active phosphorylated JNK (pJNK) and the APAP protein adducts given their prominent role in APAP hepatotoxicity. ${ }^{3,4}$ Four hours post-APAP exposure, K8 G62C/R341C mice displayed moderately increased JNK1 and robustly elevated pJNK, but not JNK2 levels (Fig. 5A). No differences were noted under basal conditions (not shown). Furthermore, 18 hours, but not 4 hours post-APAP administration, APAP protein adducts were significantly higher in $\mathrm{K} 8 \mathrm{G} 62 \mathrm{C} / \mathrm{K} 8 \mathrm{R} 341 \mathrm{C}$ mice, compared to WT K8s (Fig. 5B).

To better understand the role of K8 variants in APAP hepatotoxicity, we evaluated APAP-induced K8/K18 changes. Eighteen hours post-APAP exposure, both K8
G62C and K8 R341C mice displayed significant K8 hyperphosphorylation at S74 and S432. As a likely mechanism, pJNK levels were increased in these animals, whereas no differences were noted in phosphop38 kinase (Fig. 5C). Consistent with keratin hyperphosphorylation, mice carrying K8 variants displayed increased $\mathrm{K} 8$ solubility that was confirmed by densitometric quantification (Fig. 5D, $\mathrm{E}^{16}$ ), whereas no obvious differences in solubility were noted under basal conditions (not shown). Immunofluorescence (IF) staining showed that K8 hyperphosphorylation was most prominent in pericentral areas, where it resulted in keratin network disruption (Fig. 6A and Supporting Fig. 9). Both keratin hyperphosphorylation and keratin network 
A

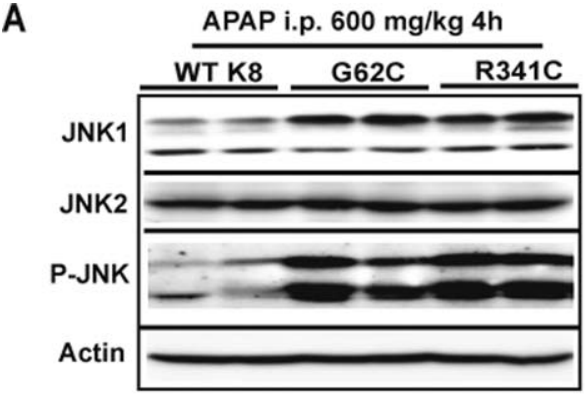

C

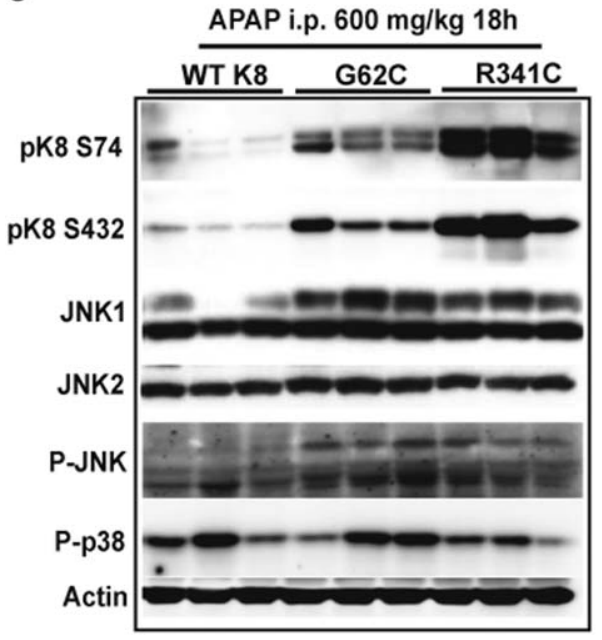

B

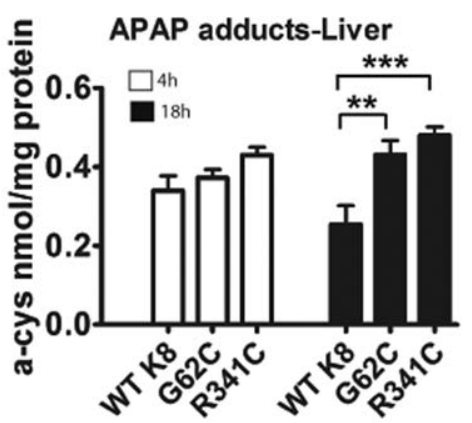

D

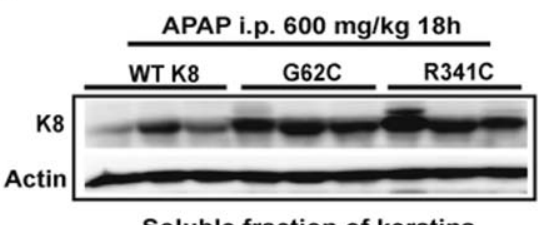

Soluble fraction of keratins

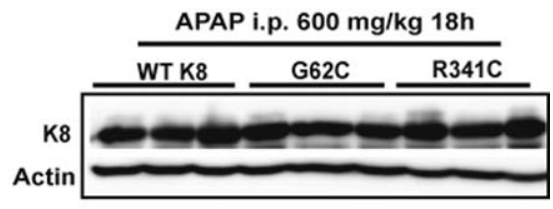

Insoluble fraction of keratins

E
Human $\mathrm{K8}$ solubility

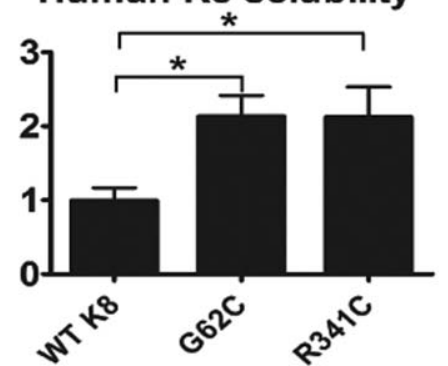

Fig. 5. Mice carrying K8 variants display activated JNK signaling, stronger formation of APAP protein adducts, K8 hyperphosphorylation, and increased K8 solubility. (A and C) Total liver lysates from APAP-exposed mice carrying either WT K8 or K8 variants were subjected to immunoblotting using antibodies to the indicated antigens. (B) Formation of APAP protein adducts was analyzed 4 and 18 hours post-APAP administration by high-performance liquid chromatography with electrochemical detection (expressed as nmol of cysteine adducts per mg of protein). (D and $E$ ) Keratin solubility in 1\% Triton X-containing buffer was assessed by immunoblotting and the ratio of soluble (upper panel) and insoluble (lower panel) K8 was quantified by densitometric analysis. K8 solubility in WT K8 mice was arbitrarily set as 1 and levels in the other genotypes represent a ratio. Actin was used as a loading control. At least 4 mice per genotype were analyzed and the results are expressed as mean \pm standard error of the mean. $* P<0.05 ; * * P<0.01 ; * * * P<0.001$. Abbreviations: i.p., intraperitoneally; $p$, phospho.

disruption were more prominent in $\mathrm{K} 8 \mathrm{G} 62 \mathrm{C} / \mathrm{R} 341 \mathrm{C}$ mice, compared to WT K8 animals (Fig. 6 and Supporting Fig. 9).

Given the reported association of K8/K18 variants with liver disease development in patients with $\mathrm{PBC},{ }^{22}$ we also analyzed the role of $\mathrm{K} 8$ variants in $\mathrm{BDL}$ induced chronic cholestatic liver injury. All animals exhibited markedly elevated bilirubin, ALT, and alkaline phosphatase (ALP) 21 days post-BDL, but no differences were noted among the genotypes (Fig. 7B-D). Moreover, the overall extent of liver injury and liver fibrosis as well as collagen mRNA levels were similar in all experimental groups (Fig. 7A,E and Supporting Fig. 10).
In summary, our data show that the presence of K8 variants predisposes to APAP hepatotoxicity, but not to cholestatic liver injury, in association with increased JNK activation, more prominent formation of protein adducts, and alterations in the K8/K18 system (Fig. 8).

\section{Discussion}

Given the established link between the presence of K8/K18 variants and the development of several human liver diseases, ${ }^{11,12}$ we generated $\mathrm{Tg}$ animals carrying common human $\mathrm{K} 8$ variants observed in Caucasians from the United States and Europe in order to delineate 
Fig. 6. Mice carrying K8 variants display pericentral keratin network disruption and K8 hyperphosphorylation post-APAP exposure. Mice overexpressing WT K8 or K8 variants were treated with APAP for 18 hours and the resulting liver sections were subjected to IF staining with K8/K18 antibody alone (a-c, $\mathrm{g}-\mathrm{i})$ or to a combination of K8/K18 (green) and the depicted phospho-specific (p) K8 antibody (red; d-f, jI). Note that animals carrying $\mathrm{K} 8$ variants display a more pronounced pericentral K8 hyperphosphorylation and keratin network disruption (the latter is highlighted by arrows), as confirmed by morphometrical quantification (B). At least 3 mice were analyzed per genotype and the results are expressed as mean \pm standard error of the mean. Scale bar $=50 \mu \mathrm{m}$. ${ }^{*} P<$ $0.05 ; * P<0.01$.
A
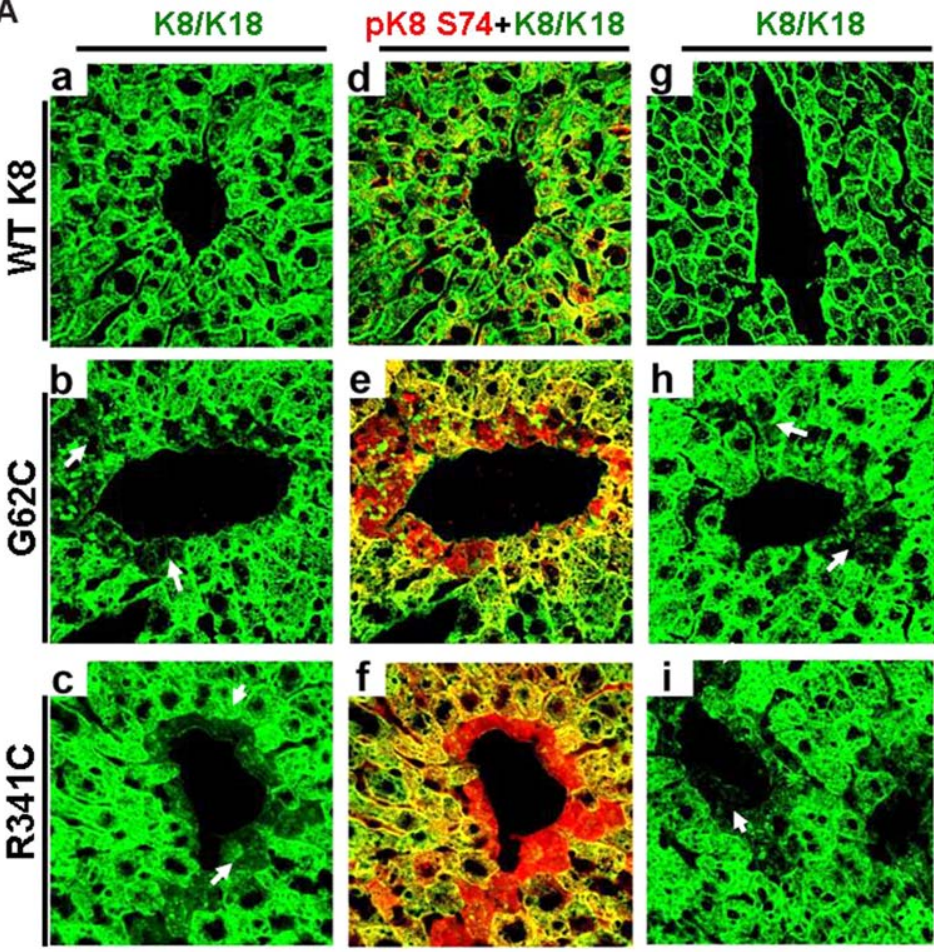

pK8 S432+K8/K18
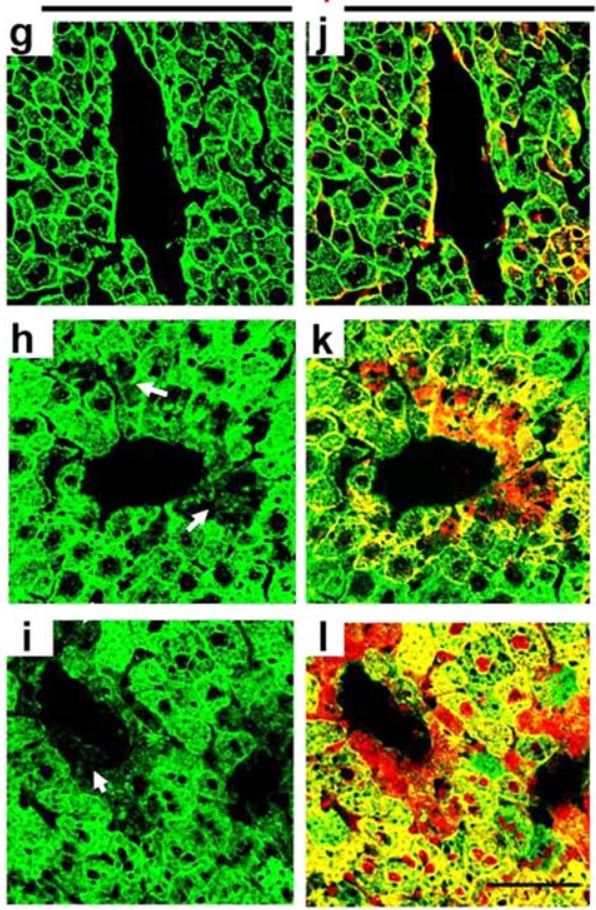

B

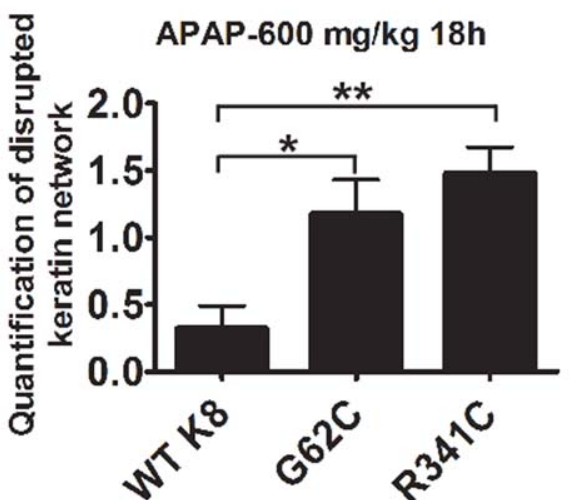

the biological significance of the variants. Under basal conditions, K8 R341H/R341C mice did not display hepatic alterations or changes in the K8/K18 filament network, which is similar to previous findings in $\mathrm{K} 8$ G62C mice (Fig. 8). In contrast, keratin filament disruption under basal conditions, as occurs in K18 R90C livers, results in hepatocyte fragility and spontaneous mild hepatitis. ${ }^{12}$ The effect of the K18 R90C mutation is not surprising given that it is located in the most conserved regions of K18 mimicking the severely disruptive keratin mutations found in epidermal genodermatoses, ${ }^{23}$ whereas K8 G62/R341, though highly conserved residues, are situated in less-conserved domains. ${ }^{11,12}$ One potential caveat of our models is the expression of the human keratins in the background of endogenous mouse keratins and the potential differences in keratin expression levels. However, human K8 appears to integrate normally into the endogenous mouse keratin filament network, which is not surprising given the $\sim 88 \%$ identity of mouse and human K8.

Post-APAP exposure, all mouse lines carrying K8 variants exhibited increased hepatotoxicity (Fig. 8), which supports the human findings that suggested a K8/K18 mutation-based predisposition toward adverse outcome of APAP-induced ALF. ${ }^{6}$ As a potential underlying mechanism, the presence of $\mathrm{K} 8$ variants resulted in a more prominent JNK activation that is instrumental in APAP-induced cell death. ${ }^{24}$ These findings are in line with previous reports that linked activation of JNK pathway to disturbances in the keratin system. For example, prolonged JNK phosphorylation was observed in keratinocyte cell lines carrying mutations in the 
A

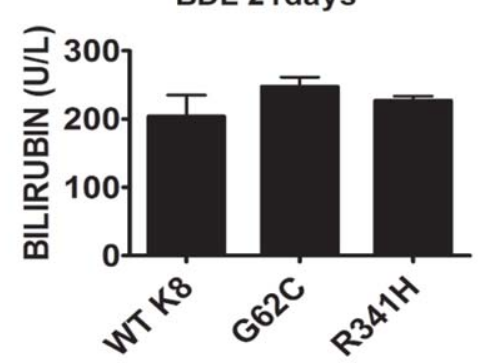

C

BDL 21days

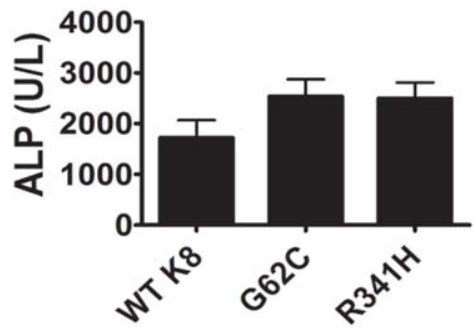

E
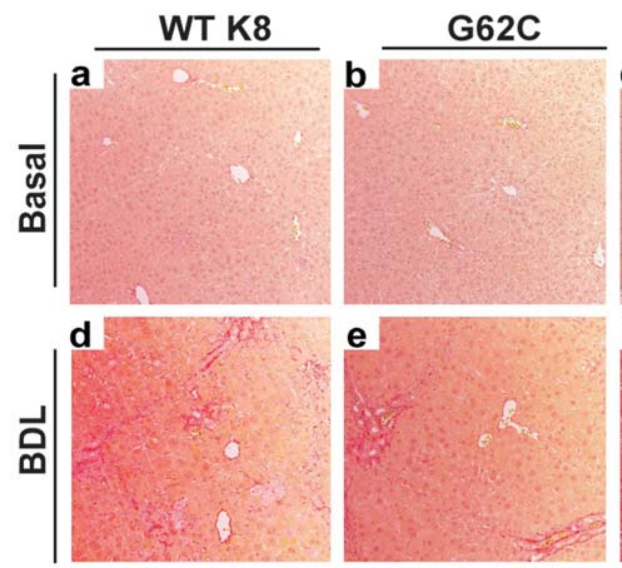

B

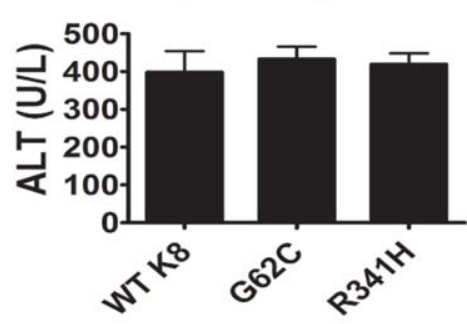

D
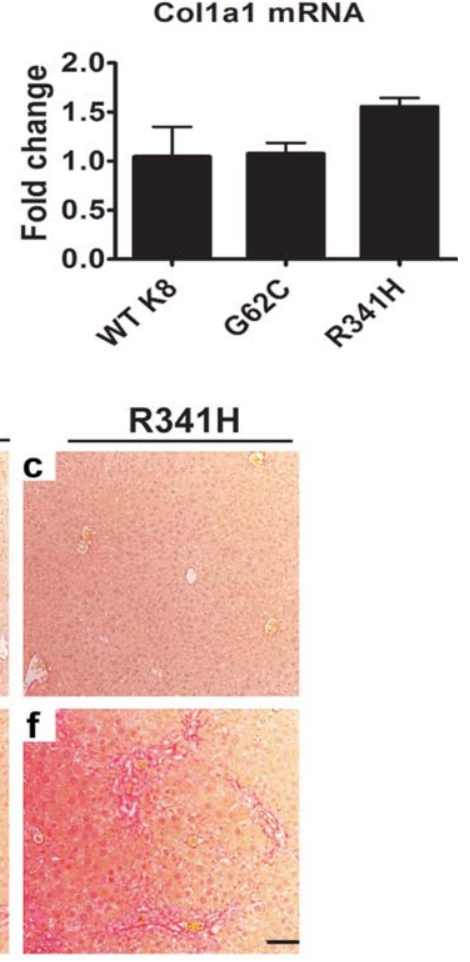

Fig. 7. Presence of K8 variants does not affect development of BDL-induced liver fibrosis. (A-C) Three weeks post$B D L$, mice displayed significantly elevated bilirubin, ALT, and ALP levels; however, no significant differences were noted among genotypes. Furthermore, in all untreated animals, bilirubin, ALT, and ALP levels were within normal range. (E) Extent of liver fibrosis in untreated animals (basal) as well as mice subjected to BDL for 3 weeks was evaluated by Picro Sirius Red staining. Note that animals overexpressing WT K8 or the depicted $\mathrm{K} 8$ variants display a similar amount of liver scarring and similar hepatic collagen mRNA levels, as determined by quantitative RT-PCR (D). L7 (mouse ribosomal protein) gene was used as an internal control. At least 4 mice were analyzed per genotype and results are expressed as mean \pm standard error of the mean. Scale bar $=100 \mu \mathrm{m}$.

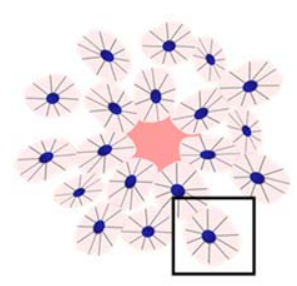

Basal condition
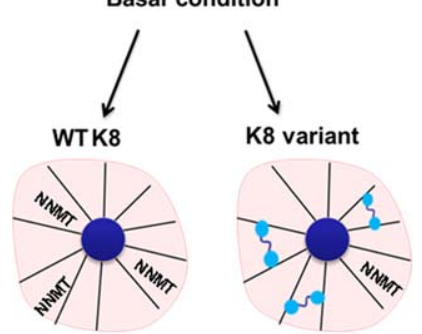

Normal keratin architecure $\mathrm{K}$ crosslinking (For Cys variants) NNMT MRNA

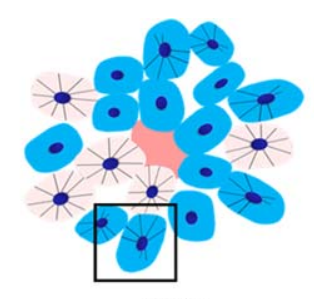

APAP
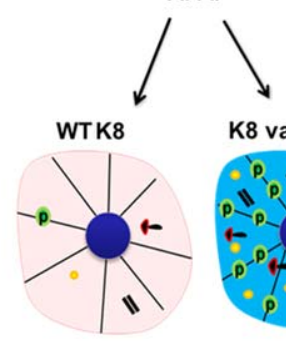

K8 variant

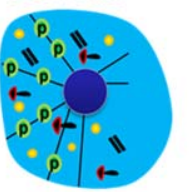

Liver damage P-JNK ^

APAP adducts -

$\mathrm{K}$ solubility

K phosphorylation

$\mathrm{K}$ disruption
- P-JNK

$\leftarrow$ APAP-adducts

\% crosslinks

p Phosphorylated K

N. Soluble Ks

- Hepatocyte

新 K network

- $K$ disruption

- Damaged hepatocyte

NNMT NNMT MRNA
Fig. 8. The schematic summarizes the findings of our study. Under basal conditions, mice carrying $\mathrm{K} 8$ variants display normal liver architecture and keratin filament network, but harbor more keratin cross-links and have lower NNMT levels than animals overexpressing WT K8. After APAP administration, mice with $K 8$ variants exhibit increased liver injury, JNK activation (i.e., P-JNK) APAP adducts formation, and alterations in K8/K18 filament organization. 
epidermal K5/K14 when exposed to osmotic stress ${ }^{25}$ or in mice ectopically expressing K10 in the basal epidermal layers. ${ }^{26}$ The increased formation of APAP protein adducts also likely contributes to the increased APAP hepatotoxicity observed in mice with $\mathrm{K} 8$ variants. Though further studies are needed to determine the exact underlying mechanisms, the decreased NNMT levels in mutant keratin-expressing mice are likely to be important. ${ }^{21,27}$ In that respect, our data demonstrated an association between K8/K18 and NNMT; however, the molecular domains involved in this association and the impact of $\mathrm{K} 8$ variants on this process remain to be characterized.

When challenged with APAP, mice overexpressing K8 variants displayed K8 hyperphosphorylation at S74 and S432. The increased K8 pS74 levels are somewhat surprising given that $\mathrm{K} 8 \mathrm{G} 62 \mathrm{C}$ substitution blunts phosphorylation at this residue (by inducing a conformation change ${ }^{28}$ ) in vitro using purified $\mathrm{p} 38$ kinase or in $\mathrm{Tg} \mathrm{K} 8$ G62C that are exposed to Fas ligand. ${ }^{17,29}$ However, K8 S74 is phosphorylated by several kinases, including p38 kinase and JNK. Therefore, the inhibitory role of the G62C substitution is likely restricted only to certain kinases. Of note, JNK is also known to phosphorylate $\mathrm{K} 8$ at $\mathrm{S} 432^{29}$ and is therefore likely responsible, at least in part, for the observed increase in K8 $\$ 432$ hyperphosphorylation.

The increased K8 phosphorylation in mice with K8 variants likely accounts for the higher $\mathrm{K} 8$ solubility as well as APAP-induced K8/K18 network reorganization, thereby overcoming the impact of $\mathrm{K} 8 \mathrm{G} 62 \mathrm{C}$ that would otherwise be expected to decrease $\mathrm{K} 8$ solubility through disulfide bond formation. ${ }^{16,17}$ Other post-translational modifications might also be important given that some of them affect keratin solubility as well. ${ }^{16}$

The more prominent disruption of pericentral keratin filament network in animals with $\mathrm{K} 8$ variants represents another factor likely contributing to the development of APAP-induced liver injury. In that respect, keratins constitute the major mechanical stabilizers of the cell, ${ }^{30}$ and the cellular fragility resulting from keratin network disruption might be particularly detrimental in the case of APAP-induced hepatocyte swelling. ${ }^{3,4,12}$ In agreement, keratin network disruption has been linked to hepatocellular ballooning in multiple liver disorders. ${ }^{31}$ Of note, keratin network disruption has been associated with susceptibility to oxidative stress as well as with impaired mitochondrial function that constitute important mechanisms promoting APAP toxicity. $3,4,32,33$

In contrast to APAP-mediated hepatotoxicity, the K8 variants did not affect the development of BDL-induced liver injury or fibrosis. This finding further underscores the previous observations showing that $\mathrm{K} 8 / \mathrm{K} 18$ variantbased predisposition to liver injury is context specific. In that respect, K18 R90C mutation increases susceptibility to Fas-induced, but not TNF- $\alpha$-induced, apoptosis and liver damage, and to thioacetamide-mediated, but not $\mathrm{CCl}_{4}$-mediated, fibrogenesis. ${ }^{34,35}$ In humans, exonic K8/K18 variants segregate with poor outcome or liver disease progression in patients with ALF, PBC, or chronic hepatitis $C$ infection, but do not associate with progression of hemochromatosis-induced liver damage. ${ }^{6,22,36,37}$

In summary, our findings provide insight and support for the disease relevance of human K8 variants in APAPassociated liver injury.

Acknowledgment: The expert technical assistance of Elke Preiß, Kristina Diepold, Claudia Laengle, Susanne Bobrovich, Linda Schaub, Giovanna Vella, and Anke Bauer is gratefully acknowledged.

\section{References}

1. Bernal W, Auzinger G, Dhawan A, Wendon J. Acute liver failure. Lancet 2010;376:190-201.

2. Fontana RJ. Pathogenesis of idiosyncratic drug-induced liver injury and clinical perspectives. Gastroenterology 2014;146:914-928.

3. Zhao L, Pickering G. Paracetamol metabolism and related genetic differences. Drug Metab Rev 2011;43:41-52.

4. Jaeschke H, McGill MR, Ramachandran A. Oxidant stress, mitochondria, and cell death mechanisms in drug-induced liver injury: lessons learned from acetaminophen hepatotoxicity. Drug Metab Rev 2012;44:88-106.

5. Watkins PB, Kaplowitz N, Slattery JT, Colonese CR, Colucci SV, Stewart PW, Harris SC. Aminotransferase elevations in healthy adults receiving 4 grams of acetaminophen daily: a randomized controlled trial. JAMA 2006;296:87-93.

6. Strnad P, Zhou Q, Hanada S, Lazzeroni LC, Zhong BH, So P, et al. Keratin variants predispose to acute liver failure and adverse outcome: race and ethnic associations. Gastroenterology 2010;139:828-835, 835.e1-3.

7. Haines RL, Lane EB. Keratins and disease at a glance. J Cell Sci 2012; 125:3923-3928.

8. Pan X, Hobbs RP, Coulombe PA. The expanding significance of keratin intermediate filaments in normal and diseased epithelia. Curr Opin Cell Biol 2013;25:47-56.

9. Szeverenyi I, Cassidy AJ, Chung CW, Lee BT, Common JE, Ogg SC, et al. The Human Intermediate Filament Database: comprehensive information on a gene family involved in many human diseases. Hum Mutat 2008;29:351-360.

10. Omary MB, Ku NO, Strnad P, Hanada S. Toward unraveling the complexity of simple epithelial keratins in human disease. J Clin Invest 2009;119:1794-1805

11. Strnad P, Paschke S, Jang KH, Ku NO. Keratins: markers and modulators of liver disease. Curr Opin Gastroenterol 2012;28:209-216.

12. Ku N, Strnad P, Zhong BH, Tao GZ, Omary MB. Keratins let liver live: Mutations predispose to liver disease and crosslinking generates Mallory-Denk bodies. Hepatology 2007;46:1639-1649.

13. Tao GZ, Strnad P, Zhou Q, Kamal A, Zhang L, Madani ND, et al. Analysis of keratin polypeptides 8 and 19 variants in inflammatory bowel disease. Clin Gastroenterol Hepatol 2007;5:857-864. 
14. Strnad P, Usachov V, Debes C, Grater F, Parry DA, Omary MB. Unique amino acid signatures that are evolutionarily conserved distinguish simple-type, epidermal and hair keratins. J Cell Sci 2011; 124:4221-4232.

15. Ku NO, Gish R, Wright TL, Omary MB. Keratin 8 mutations in patients with cryptogenic liver disease. N Engl J Med 2001;344:1580-1587.

16. Snider NT, Omary MB. Post-translational modifications of intermediate filament proteins: mechanisms and functions. Nat Rev Mol Cell Biol 2014;15:163-177.

17. Ku NO, Omary MB. A disease- and phosphorylation-related nonmechanical function for keratin 8. J Cell Biol 2006;174:115-125.

18. Krauss S, Franke WW. Organization and sequence of the human gene encoding cytokeratin 8. Gene 1990;86:241-249.

19. Magin TM, Schroder R, Leitgeb S, Wanninger F, Zatloukal K, Grund C, Melton DW. Lessons from keratin 18 knockout mice: formation of novel keratin filaments, secondary loss of keratin 7 and accumulation of liver-specific keratin 8-positive aggregates. J Cell Biol 1998;140: 1441-1451.

20. Nakamichi I, Toivola DM, Strnad P, Michie SA, Oshima RG, Baribault H, Omary MB. Keratin 8 overexpression promotes mouse Mallory body formation. J Cell Biol 2005;171:931-937.

21. Aksoy S, Szumlanski CL, Weinshilboum RM. Human liver nicotinamide $\mathrm{N}$-methyltransferase. cDNA cloning, expression, and biochemical characterization. J Biol Chem 1994;269:14835-14840.

22. Zhong B, Strnad P, Selmi C, Invernizzi P, Tao GZ, Caleffi A, et al. Keratin variants are overrepresented in primary biliary cirrhosis and associate with disease severity. Hepatology 2009;50:546-554.

23. Coulombe PA, Lee $\mathrm{CH}$. Defining keratin protein function in skin epithelia: epidermolysis bullosa simplex and its aftermath. J Invest Dermatol 2012;132:763-775.

24. Seki E, Brenner DA, Karin M. A liver full of JNK: signaling in regulation of cell function and disease pathogenesis, and clinical approaches. Gastroenterology 2012;143:307-320.

25. D’Alessandro M, Russell D, Morley SM, Davies AM, Lane EB. Keratin mutations of epidermolysis bullosa simplex alter the kinetics of stress response to osmotic shock. J Cell Sci 2002;115:4341-4351.

26. Santos M, Perez P, Segrelles C, Ruiz S, Jorcano JL, Paramio JM. Impaired NF-kappa B activation and increased production of tumor necrosis factor alpha in transgenic mice expressing keratin K10 in the basal layer of the epidermis. J Biol Chem 2003;278:13422-13430.

27. Nelson SD, Forte AJ, McMurtry RJ. Decreased toxicity of the Nmethyl analogs of acetaminophen and phenacetin. Res Commun Chem Pathol Pharmacol 1978;22:61-71.
28. Tao GZ, Nakamichi I, Ku NO, Wang J, Frolkis M, Gong X, et al. Bispecific and human disease-related anti-keratin rabbit monoclonal antibodies. Exp Cell Res 2006;312:411-422.

29. Ku NO, Lim JK, Krams SM, Esquivel CO, Keeffe EB, Wright TL, et al. Keratins as susceptibility genes for end-stage liver disease. Gastroenterology 2005;129:885-893.

30. Chung BM, Rotty JD, Coulombe PA. Networking galore: intermediate filaments and cell migration. Curr Opin Cell Biol 2013;25:600-612.

31. Lackner C, Gogg-Kamerer M, Zatloukal K, Stumptner C, Brunt EM, Denk H. Ballooned hepatocytes in steatohepatitis: The value of keratin immunohistochemistry for diagnosis. J Hepatol 2008;48:821-828.

32. Zhou Q, Ji X, Chen L, Greenberg HB, Lu SC, Omary MB. Keratin mutation primes mouse liver to oxidative injury. Hepatology 2005; 41 : 517-525.

33. Tao GZ, Looi KS, Toivola DM, Strnad P, Zhou Q, Liao J, et al. Keratins modulate the shape and function of hepatocyte mitochondria: a mechanism for protection from apoptosis. J Cell Sci 2009;122:38513855.

34. Ku NO, Soetikno RM, Omary MB. Keratin mutation in transgenic mice predisposes to Fas but not TNF-induced apoptosis and massive liver injury. HEPATOLOGY 2003;37:1006-1014.

35. Strnad P, Tao GZ, Zhou Q, Harada M, Toivola DM, Brunt EM, Omary MB. Keratin mutation predisposes to mouse liver fibrosis and unmasks differential effects of the carbon tetrachloride and thioacetamide models. Gastroenterology 2008;134:1169-1179.

36. Strnad P, Lienau TC, Tao GZ, Ku NO, Magin TM, Omary MB. Denaturing temperature selection may underestimate keratin mutation detection by DHPLC. Hum Mutat 2006;27:444-452.

37. Strnad P, Kucukoglu O, Lunova M, Guldiken N, Lienau TC, Stickel F, Omary MB. Non-coding keratin variants associate with liver fibrosis progression in patients with hemochromatosis. PLoS One 2012;7: e32669.

Author names in bold designate shared co-first authorship.

\section{Supporting Information}

Additional Supporting Information may be found at onlinelibrary.wiley.com/doi/10.1002/hep.27891/suppinfo. 